\title{
Modeling of Dust Deposition Affecting Transmittance of PV Modules
}

\author{
Jingshu Wang, Hengxiang Gong, and Zheng Zou
}

\begin{abstract}
The dust deposition significantly influences the energy gained from the solar power system. The dust deposition of PV modules is decided many factors, such as weather, location, tilt angle, and so on. This paper focuses on the influence factors of the PV modules. In order to realize the relationship between the dust deposition and the sunlight transmittance of the solar PV module, improved models considering the effect of incident angle and tilt angle are developed based on the overlay model. Then simulation with the improved models is conducted to analyze the characteristics of each model.
\end{abstract}

Index Terms-Dust deposition, transmittance, incident angle, tilt angle.

\section{INTRODUCTION}

As the core component of the solar power system, the solar $\mathrm{PV}$ panel is long-running, so the effect of the dust deposition on the system efficiency is significant. The dust deposition of PV module directly leads to the reduction of the solar radiation received and the decrease of the energy gained from the solar power system. The sources of the dust including the tiny particle formed by the action of sand, soil and wind, the blowing dust due to the industry, building, and transportation, and the biomass of animals and plants [1]-[3]. The longer the solar power system is running, the more the dust deposition will be, therefore, the lower transmittance and the less generated energy is caused. With the other conditions being equal, the smaller tilt angle of the PV module leads to the more dust deposition and the less solar radiation received.

Although the effect of dust on a single PV cell is noteless, the power loss owning to the dust of the large-scale PV system is great [4]. The research found that in the dry period, efficiency loss resulted from the dust deposition was as much as $15 \%$, and the average annual power generation efficiency reduced $6 \%$.

In recent years, more and more researchers pay attention to the influence of the dust deposition on the efficiency of the PV module. Most scholars exposed the PV module in different environments, analyzed the effect of the dust deposition by the comparison of experiment results [5]-[7].

Manuscript received October 10, 2015; revised March 10, 2016. This work was supported in part by the science and technology planning program of Chongqing, No. cstc2014yykfB0037, and Scientific and Technological Research Program of Chongqing Municipal Education Commission, No. KJ1400936.

Jingshu Wang and Zheng Zou are with the School of Mechanical Engineering, Chongqing University of Technology, Chongqing, China (e-mail: donot@cqut.edu.cn, z.zou@cqut.edu.cn).

Hengxiang Gong is with the School of Photoelectric Information, Chongqing University of Technology, Chongqing, China (e-mail: gonghx@cqut.edu.cn).
However, because the climatic environment varied from place to place, there were large differences among the research results.

The mechanism of the dust deposition is another research field. Many experts considered the dust with different size, different component, and different moisture and modeled the process of the deposition [8]-[11]. But so far, there were rarely research focuses on the relationship between the dust deposition and the sunlight transmittance of the solar PV module.

This paper concentrates on the modeling of dust deposition affecting transmittance of PV modules. In the following sections, firstly, an overlay model of the dust deposition has been developed. Then, the improved incident angle model and tilt angle model has been derived. Last, the comparison among the different models was done based on the simulation results.

\section{OVERLAy MODEL}

\section{A. Basic Model}

NASA (National Aeronautics and Space Administration) researched the principle of dust deposition on the Mars and the moon, considering the sources, the property, and the spread of the dust. Based on the influence of the dust deposition on the solar PV modules, NASA developed the overlay model to describe the influence [12].

The overlay model is feasible under the following theoretical assumptions: (a) All the dust particles are spherical. (b) The ratio of the area dust covered to the panel area is equal to the ratio of the transmittance with dust to the transmittance without dust.

Besides the assumptions, the total area of the panel is indicated by $A$, and the area of single dust particle is $\alpha$. Then, the ratio of the area without dust is $1-\alpha / A$. If the number of dust particles is $N$, the ratio of the area without dust goes to $(1-\alpha / A)^{N} . \gamma$ is used as the average transmission of single layer dust.

Considering the dust may be overlapping, the following equation is derived as Equation (1). This is the most important assumption in this model. $\left(1-\frac{\gamma \alpha}{A}\right)^{N}$ means one dust may overlap another and affect the light transmittance. If the equation is $\left(1-N \frac{\gamma \alpha}{A}\right)$ means just the cumulative effect of the dust is considered. When parameter $N$ is large enough, $\left(1-N \frac{\gamma \alpha}{A}\right)$ may be negative and it is unphysical. 


$$
\frac{\tau_{2}}{\tau_{1}}=\frac{F}{F_{0}}=\left(1-\frac{\gamma \alpha}{A}\right)^{N}
$$

where $\tau_{1}$ and $\tau_{2}$ respectively represent the transmission before and after dust deposition of PV modules; $\tau_{2} / \tau_{1}$ indicates the relative transmission; $F_{0}$ and $F$ respectively represent the area before and after dust deposition of PV modules.

\section{B. Applied Model}

As the parameter $N$, namely, the number of the dust particles is difficult to measure, an applied overlay model has been developed.

Let us define the variable $j$ as

$$
j=\frac{A}{\gamma \alpha} .
$$

Then, the number of the dust particles is

$$
N=\frac{M}{\rho V}=\frac{3 M}{\rho^{4} \pi R^{3}}=\frac{3 M j \gamma}{4 \rho A R},
$$

where $M$ is the total mass of the dust particles, $\rho$ is the average density of the particle, $V$ and $R$ respectively represent the total volume and average radius of the dust particles.

When the PV panel is covered by dust particles with number of $N$, the area of the PV panel without dust is described as

$$
F=F_{0}\left[\left(1-\frac{1}{j}\right)^{j}\right]^{\frac{3 M \gamma}{4 \rho R A}}
$$

When

$$
\frac{A}{\alpha} \rightarrow \infty,\left(1-\frac{1}{j}\right)^{j} \rightarrow \exp (-1)
$$

We can get

$$
F=F_{0} \exp \left(-\frac{3 M \gamma}{4 \rho R A}\right)
$$

Ignore the scattering of the dust, the relative transmittance with the mass of dust being $M$ is

$$
\frac{\tau_{2}}{\tau_{1}}=\exp \left(-\frac{3 M \gamma}{4 \rho R A}\right) \text {. }
$$

Equation (2) is the applied overlay model. According to (2), the relative transmittance of PV module depends on the mass, the transmission, the density, and the radius of the dust particle. Meanwhile, the transmission and the density of general dust are basically similar. Consequently, the relative transmittance relies on the mass and the average radius of dust.

\section{IMPROVED MODEL}

\section{A. Incident Angle Model}

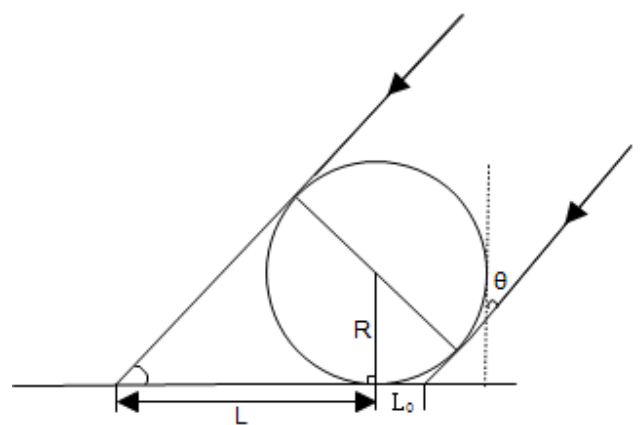

Fig. 1. The diagram of the incident angle of the dust.

Professor Zang et al. added the incident angle into the overlay model and derived an incident angle model [13]. As shown in Fig. 1, the incident angle is presented by $\theta$, when $\theta$ is not equal to zero, the shadow of the dust particle is oval. The major and minor of axes are $L+L_{0}$ and $R$, respectively. So the area of the shadow is

$$
F(\theta)=\pi R \frac{L+L_{0}}{2}=\frac{\pi R^{2}}{\cos \theta}=\frac{\alpha}{\cos \theta} .
$$

Combining with (2), the relative transmittance considering the incident angle is

$$
\frac{\tau_{2}(\theta)}{\tau_{1}(\theta)}=\exp \left(-\frac{3 M \gamma}{4 \rho R A \cos \theta}\right)
$$

\section{B. Tilt Angle Model}

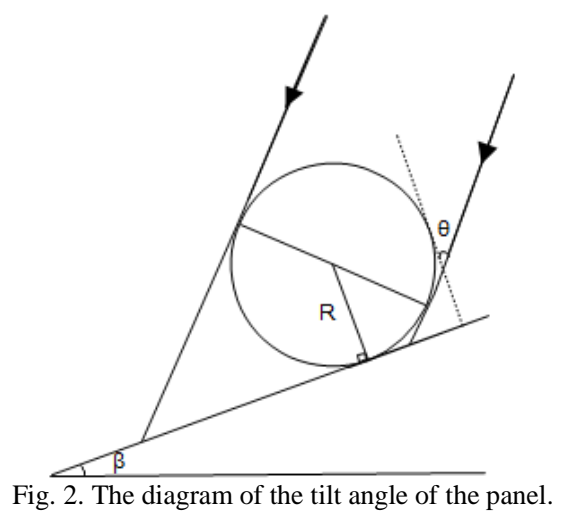

Owning to the gravity, it is easy to understand that the magnitude of the tilt angle directly effects the performance of the dust deposition. The dust quantity with tilt angle being $0^{\circ}$, namely the panel is horizontal, is much more than the quantity with tilt angle being $90^{\circ}$, namely vertical. The diagram of the tilt angle is shown in Fig. 2.

Recent years, many researchers experimented with different tilt angle and measured the efficiency of the solar system. The results proved that the tilt angle of the PV module directly influenced the dust deposition.

Sayigh et al. experimented five PV modules with different tilt angles [14]. With the other conditions being the same, the reduction rate of the transmission after 38 days is shown in Table I. 
TABLE I: THE EXPERIMENT RESULTS WITH DifFERENT TILT ANGLES

\begin{tabular}{llllll}
\hline Tilt angle & $0^{\circ}$ & $15^{\circ}$ & $30^{\circ}$ & $45^{\circ}$ & $60^{\circ}$ \\
\hline Reduction rate & $64 \%$ & $48 \%$ & $38 \%$ & $30 \%$ & $17 \%$ \\
\hline \hline
\end{tabular}

Therefore, the parameter of tilt angle, ranges from $0^{\circ}$ to $90^{\circ}$, should be considered in the improved model. The actual tilt angle is determined by the latitude of the geographic location, which is different from area to area. The character $\beta$ is used to indicate the tilt angle and the function $f(\beta)$ presents the influence. So the improved tilt angle model is

$$
\frac{\tau_{2}(\theta)}{\tau_{1}(\theta)}=\exp \left(-\frac{3 M \gamma}{4 \rho R A \cos \theta}\right) f(\beta)
$$

The tilt angle affects the dust deposition through the gravity. In the actual process, the wind, the climatic environment, the humidity and many other factors also affect the dust deposition. Therefore, it is extremely complex to analyze theoretically [15], [16].

The data fitting is employed to simplify the relationship between the tilt angle and the reduction rate. Based on the data supported by Sayigh, the least square method is applied. The result is presented in Fig. 3, and the relationship is described as

$$
f(\beta)=K\left(-0.5109 \beta^{3}+0.8858 \beta^{2}+0.8162 \beta+0.6404\right)
$$

where the $K$ is a variable parameter based on different conditions.

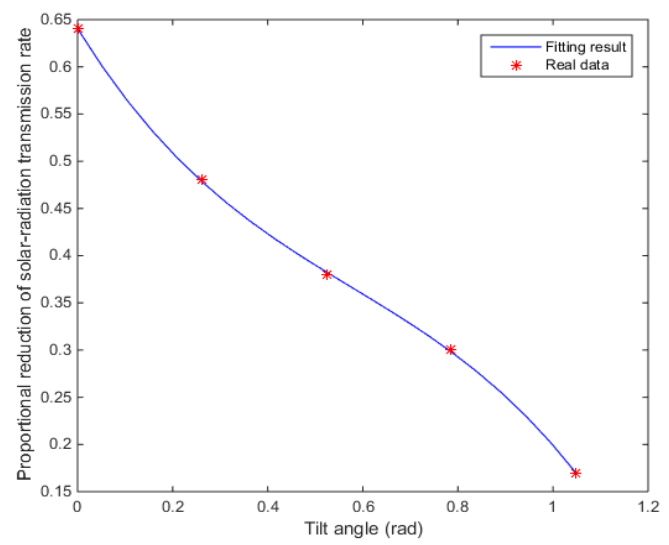

Fig. 3. The result of the data fitting.

\section{Martin Model}

Considering only the influence of the incident angle and ignoring the effect of dust, a simple empirical formula was developed by ASHRAE, as equation (5).

$$
\frac{\tau_{2}(\theta)}{\tau_{1}(\theta)}=1-b\left(\frac{1}{\cos \theta}-1\right)
$$

The $b$ is an empirical parameter based on the type of the solar panel, and it is equal to 0.07 commonly [17].

When the influence of dust is considered, equation (5) changes to

$$
\frac{\tau_{2}(\theta)}{\tau_{1}(\theta)}=1-\frac{\exp \left(-\frac{\cos \theta}{\eta}\right)-\exp \left(-\frac{1}{\eta}\right)}{1-\exp \left(-\frac{1}{\eta}\right)} \frac{\tau_{2}(0)}{\tau_{1}(0)}
$$

In (6), $\eta$ is the empirical parameter according to the pollution level of the solar panel, and it is equal to 0.07 commonly [18], [19]. $\frac{\tau_{2}(0)}{\tau_{1}(0)}$ means the relative transmission with the incident angle being 0 .

\section{SimUlation}

\section{A. Simulation with Changed Incident Angle}

In order to description conveniently, the models expressed by equations (3) and (4) call as "incident angle model" and "tilt angle model", respectively. The model presented by equation (5) ignores the effect of dust, so it calls as "no dust model". The model presented by (6) calls as "Martin model".

The simulation with changed incident angle is conducted with incident angle model, no dust model, and Martin model. In this simulation, the parameter $\rho$ is equal to $2000 \mathrm{~kg} / \mathrm{m}^{3}$ and $\mathrm{R}$ is ranged from $0.2 \times 10^{-6} \mathrm{~m}$ to $40 \times 10^{-6} \mathrm{~m}$, while average transmission $\gamma$ is equal to 0.87 , and the total mass of dust $M$ is assumed to be $4 \times 10^{-3} \mathrm{~kg}[20]$.

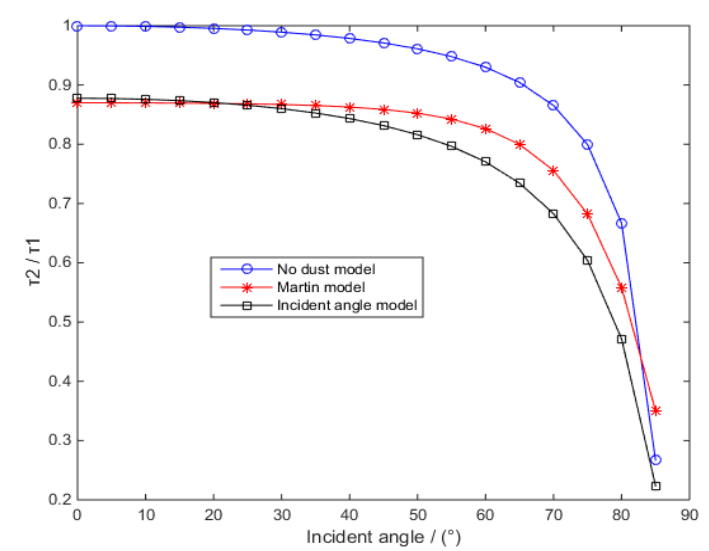

Fig. 4. The simulation result with changed incident angle.

As shown in Fig. 4, the simulation result indicates that the relative transmissions of both incident angle model and Martin model are less than the one of no dust model. This result is conform to the real situation. The decreasing trend of all three models appears slowly first and fast afterwards. The demarcation point is about incident angle being $60^{\circ}$.

The relative transmission of incident angle model basically agree well Martin model when the incident angle being less than $30^{\circ}$. While the incident angle is larger than $30^{\circ}$, the relative transmission of incident angle model is less than Martin model, the maximum difference of which is up to $10 \%$.

\section{B. Simulation with Changed Tilt Angle}

The simulation with changed incident angle ignores the effect of tilt angle. This section the simulation with invariable incident angle and changed tilt angle is conducted. The simulation result of tilt angle model is shown in Fig. 5. 


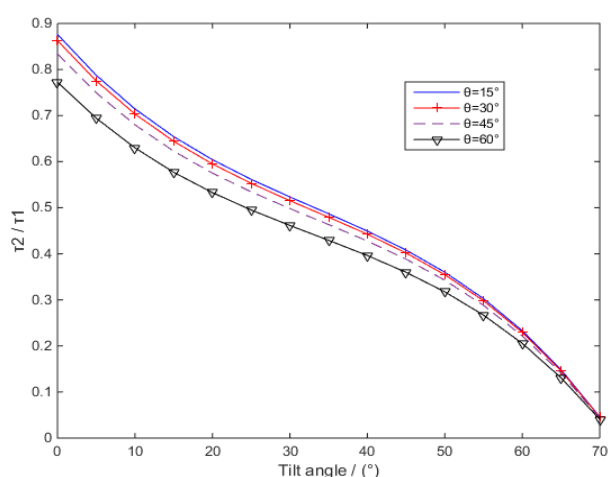

Fig. 5. The simulation result with changed tilt angle.

The simulation result indicates that whatever the incident angle, the relative transmission decreases sharply with the increase of the tilt angle. There is little difference between the simulation results with incident angle being $15^{\circ}$ and $30^{\circ}$. With particular small incident angle, the decrease of the relative transmission is more sharply. The decreasing trend of the relative transmission becomes a little slow while the incident angle increases from $15^{\circ}$ to $60^{\circ}$.

As the simulation is based on the experiment results supported by Sayigh, which was implemented in Kuwait, the simulation result is not completely suitable for the other areas However, the change rule of the relative transmission with the variation of incident angle and tilt angle has universal significance.

\section{MODEL APPLICATION}

As the incident angle model and the tile angle model can analyze the influence of the dust deposition on the system transmittance effectively, they have widespread applications in the field of photovoltaic generating system. The applications are described in two aspects.

At first, during the design stage of the photovoltaic system, the dust sources will be analyzed based on the local environment. A new Table I suited local conditions will be developed by experiments. Then, the incident angle model and tile angle model can be applied to evaluate the influences of dust deposition on the transmittance of PV modules, which must be considered in parameters design of the photovoltaic system.

Secondly, when running a photovoltaic system, the incident angle model and the tile angle model can be used to forecast the influences of dust deposition on the transmittance of PV modules. The power loss due to dust can be calculated and an effective cleaning cycle of PV modules will be determined.

In general, both in design and operation of the photovoltaic system, the models developed in this paper are useful.

\section{DISCUSSION AND CONCLUSION}

This paper focuses on the modeling of dust deposition affecting transmittance of PV modules. Based on the overlay model supported by NASA, the improved incident angle model and tilt angle model has been derived to analyze more completely. The simulations with changed incident angle and changed tilt angle have been conducted to compare the improved models.
In the future, the actual experiment is being considered to verify the simulation results and provide a basis for the model updating.

\section{REFERENCES}

[1] D. B. Chen, D. S. Li, J. Shi et al., "The experiment and analysis of the dust and the poling shadow on the surface of solar panel," Solar Energy, vol. 9, pp. 39-41, 2011

[2] F. Wang, Y. Q. Zhang, and S. Cai, "Research of the dust in Xi'an on distributed photovoltaic power station," Solar Energy, vol. 13, pp. 38-40, 2013.

[3] X. Chen, C. Wu, H. Li, X. Feng, and Z. Li, "PV fouling detecting system based on neural network and fuzzy logic," Intelligent Computing iin Smart Grid and Electrical Vehicles, vol. 463, pp. 368-377, 2014.

[4] F. Zhang, J. B. Bai, Y. Z. Hao et al., "Influence of the dust of the solar panel surface on the power performance," East China Electric Power, vol. 41, no. 2, pp. 0363-0366, 2013.

[5] S. A. M. Said, "Effect of dust accumulation on performance of thermal and photovoltaic flat-plate collectors," Applied Energy, vol. 37, no. 1, pp. 73-84, 1990.

[6] M. Mani and R. Pillai, "Impact of dust on solar photovoltaic performance — research status, challenges and recommendations," Renewable and Sustainable Energy Reviews, vol. 14, no. 9, pp. 3124-3131, 2010.

[7] S. B. Neil, S. M. Robert, C. Charlslee et al., "Understanding the effects of sand and dust accumulation on photovoltaic modules," Renewable Energy, vol. 48, pp. 448-452, 2012.

[8] V. Badescu, "Weather influence on PV solar cells operation on Mars," Mars, pp. 67-82, 2009.

[9] V. Katsioulis, E. Karapidakis, M. Hadjinicolaou et al., "Wireless monitoring and remote control of PV systems based ono the Zigbee protocol," Technological Innovation for Sustainability, vol. 349, pp. 297-304, 2011.

[10] A. A. Hegazy, "Effect on dust accumulation on solar transmittance through glass covers of plate-type collectors," Renewable Energy, vol. 22, no. 4, pp. 525-540, 2001.

[11] J. K. Kaldellis, K. J. Chalvatzis, and G. C. Spyropoulos, "Transboundary air pollution balance in the new integrated European environment," Environmental Science and Policy, vol. 10, no. 7, pp. 725-733, 2007.

[12] C. M. Katzan and J. L. Edwards, "Lunar dust transport and potential interactions with power system components," NASA CR-4404, 1991.

[13] J. B. Zang, Y. W. Wang, and X. D. Wang, "Computation model and experiment of dust deposition affecting transmittance of photovoltaic module," Acta Energiae Solaris Sinica, vol. 35, no. 4, pp. 624-629.

[14] A. Sayigh, S. Al-Jandal, and H. Ahmed, "Dust effect on solar flat surfaces devices in Kuwait," in Proc. the Workshop on the Physics of Non-conventional Energy Sources and Materials Science for Energy, Triest, Italy, 1985, pp. 353-367.

[15] J. Cano, "Effect of tilt angle on soiling," Phoenix: Arizona State University, 2011.

[16] H. K. Elminir, A. E. Ghitas, R. H. Hamid et al., "Effect of dust on the transparent cover of solar collectors," Energy Conversion and Management, vol. 47, pp. 3192-3203, 2006.

[17] ASHRAE, "Methods of testing to determine the thermal performance of solar collectors," New York: American Society of Heating, Refrigeration, and Air Conditioning Engineers, pp. 93-77, 1977.

[18] N. Martin and J. Ruiz, "Calculation of the PV modules angular losses under field conditions by means of ananalytical model," Solar Energy Materials and Solar Cells, vol. 70, pp. 25-38, 2001.

[19] A. Luque and S. Hegedus, Handbook of Photovoltaic Science and Engineering, Chichester: Wiley, 2003.

[20] G. S. Meng, D. D. Gao, S. Wang et al., "Mechanics modeling of dust particle on solar panel surface in desert environment," Transactions of the Chinese Society of Agricultural Engineering, vol. 30, no. 16, pp. 221-229, 2014.

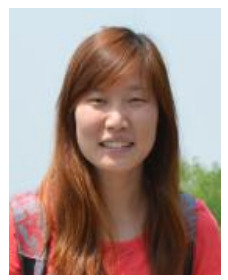

Jingshu Wang was born in Anhui, China, in 1988 She received the Ph.D. degree of precision instruments and machinery form the University of Science and Technology of China in 2013.

From July 2013 to present, she belonged to the Mechanical Engineering School of Chongqing University of Technology as a lecturer. Her present research focus on the photovoltaic system and the system modeling. 


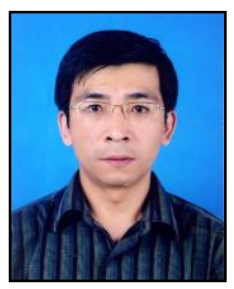

Hengxiang Gong was born in Lanzhou city, Gansu, China, on Jan. 2, 1971. He received the Ph.D. degree in physics from Lanzhou University, China in 2002. $\mathrm{He}$ is currently a lecturer in the School of Optoelectronic Information, Chongqing University of Technology, China. His current research interests are photovoltaic power generation technology application in southwest China.

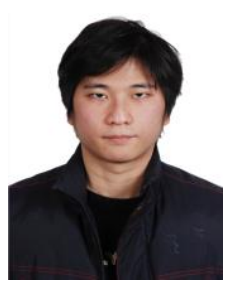

Zheng Zou was born in Chongqing, China, on May 4 1985. He received the Ph.D. degree in mechanic engineering from University of Queensland, Australia in 2014. He is currently a lecturer in the School of Mechanical Engineering, Chongqing University of Technology, China. His current research interests are solar thermal application and air cooling technology. 